\title{
Rururbanidad cyborg. Aproximación tecnocientífica a un modelo graduado de lo rural
}

\author{
J.R. Coca* ${ }^{*}$ y J.A. Valero-Matas ${ }^{* *}$ \\ * Dpto. de Sociología y Trabajo Social. Universidad de Valladolid. Campus "Duques de Soria". C.P. \\ 42004, Soria (España) \\ ** Dpto. de Sociología y Trabajo Social. Universidad de Valladolid. Campus de "La Yutera", Avda. de \\ Madrid, 44. 34004, Palencia (España)
}

\section{Resumen}

La globalización de la tecnociencia ha producido una gran cantidad de cambios sociales. Entre ellos podemos destacar el de la cyborgización del mundo rural. En el presente artículo se expone este proceso, sus consecuencias y las dificultades de análisis subyacente a través del estudio de la población de Santa Uxía de Riveira. Por último se muestra un modelo de análisis graduado que permite estudiar la nueva configuración de la ruralidad y la urbanidad.

Palabras clave: Ruralidad, tecnociencia, cyborg.

\begin{abstract}
Cyborg rururbanity. Technoscientific approach to a rural graduate model

The globalization of technoscience produced a great amount of social changes. Among them we can highlight the cyborgization of rural word. In this article we expose this process, its consequences and the underlying analyzed difficulties through the Santa Uxía de Riveira population's study. Last we show a graduate analysis fuzzy model that it allow study the new configuration of the rurality and the urbanity.
\end{abstract}

Key words: Rurality, technoscience, cyborg.

\section{Introducción}

En los últimos años se ha venido produciendo un fenómeno de gran importancia que ha condicionado el desarrollo global de la humanidad: la globalización. Dicho proceso ha cambiado la cara de la economía, de la sociedad y de la política, entre otros. Los actuales procesos de globalización, unidos a la transformación de la condición histórica de la que hablaba Lyotard en el paso de la modernidad a la postmodernidad, han devenido en una dilución de los límites convencionales en los espacios sociales. En este sentido es factible afirmar que cada vez es más habitual tener cierta incapacidad en categorizar las zonas geográficas en la vive parte de nuestra sociedad. El ejemplo palpable de este hecho nos lo encontramos en el ámbito rural/urbano en el que constantemente nos encon-

1. Autor para correspondencia: juancoca@soc.uva.es http://dx.doi.org/10.12706/itea.2013.010 
tramos con regiones rurales urbanizadas, zonas urbanas ruralizadas, etc. (Baigorri, 1995) En el presente trabajo buscamos, desde una perspectiva teórico-reflexiva, incorporar una nueva manera de enfrentarse a esta nueva realidad que nos encontramos en el ámbito rural a través de las herramientas que nos aporta la lógica fuzzy.

Entre lo rural y lo urbano

El contexto en el que nos situamos ha tomado cuerpo a partir del análisis de los distintos tipos de desarrollo territorial y como éste ha generado el concepto de urbanización difusa en oposición al de urbanización concentrada propia de la revolución industrial (Ferrás, 2000a). Dicha conceptualización implica el incremento del espacio clásico de urbanización a través de una expansión laxa y, si se nos permite, graduada/difusa de la urbanización. Este nuevo proceso social, geográfico y económico mantiene una clara vinculación con las denominadas áreas suburbanas y con las Urban Fringe: áreas de influencia directa de una zona urbana (Bryant, Russwurmm, McLeIlan, 1982). Recuérdese, como bien ha afirmado Horacio Capel (1975) que lo urbano es una realidad cambiante que trae consigo dificultades de delimitación a causa de esta naturaleza mutante del espacio urbano, pasando de ser un espacio administrativo, político y económico (a través del mercado), a incorporar funciones industriales. Actualmente, lo urbano se ha visto nuevamente transformado por la incorporación de las tecnologías de la información y comunicación dando lugar a la aparición de las áreas suburbanas. Estas nuevas zonas surgen de una nueva manera de habitar el espacio a partir de una debilitación de los lindes entre el lugar de trabajo y el lugar de residencia.

"En la difusión desconcentrada de la urbanización intervienen los procesos de suburbanización y de contraurbanización que tienen por denominador común el hecho de que su presencia implica movimientos desconcentradores de población en los asentamientos urbanos desde el centro hacia la periferia y, también, en su organización jerárquica desde los que tienen mayor número de habitantes hasta los de menos habitantes" (Ferrás, 2000a).

Este fenómeno de desconcentración se asemeja enormemente al de contraurbanización, aunque presenta graves problemas de análisis. Sobre todo debido a que, como afirma Mitchell (2004), este último es un concepto caótico que está basado en la definición de Berry (1976) quien afirma que la contraurbanización implica un proceso de desconcentración poblacional gracias a una migración de las personas de regiones más concentradas a zonas con menor densidad poblacional. Es decir, a raíz de la actual configuración del sistema social se ha producido un cambio sustancial en la dispersión de la población pasando de estar concentrada en grandes núcleos urbanos a deslocalizarse hacia zonas periféricas a éstos. Ello implica un obligado uso de medios de transporte de la periferia urbana hacia los núcleos poblacionales centrales, lo que implica un reto en la gestión del transporte, un incremento en la capacidad de movilidad humana y un creciente impacto del traslado constante en el medio ambiente. Por otro lado es constatable que estas zonas pasan a presentar unas características intermedias entre el mundo típicamente urbano y el rural, lo que implica una mayor diversidad poblacional proveniente de la participación de diversos grupos sociales (Ferrás, 2000b). Por lo tanto, la contraurbanización tiene una composición variada que puede llegar a implicar el poblamiento de regiones, cercanas a los grandes núcleos urbanos, previamente poco pobladas o en proceso de despoblamiento. Por todo ello, coincidimos con Ferrás (2000a), y contra lo expuesto por Berry (1976), que la actual contraurbanización es ambigua, algo con- 
fusa e implica el surgimiento de nuevos procesos para los que los científicos sociales necesitan desarrollar nuevas herramientas de análisis. Lo dicho implica que se esté materializando un fenómeno de licuefacción del mundo urbano al desplazarse éste hacia áreas rurales disolviendo la clásica dicotomía entre mundo rural y urbano. Además, incluso podemos afirmar que lo que también está sucediendo en un nuevo proceso que se ha dado en llamar como la cyborgización de lo rural (Coca y Valero, 2012). Esta nueva conceptualización viene a consistir en la artefactualización de todo el mundo rural y del desarrollo del mismo a través de la introducción de las TIC, de las biotecnologías, etc.

Establecer una delimitación entre lo que es y lo que no es espacio rural, ruralidad, tiene gran importancia para poder establecer que es y que no el espacio urbano. La relevancia de tal diferenciación radica en el hecho de que las políticas sociales, las políticas de transporte, las necesidades de la población, los posibles desarrollos socio-económicos, etcétera son diferentes y obliga a tener una distinción muy clara entre ambos entornos.

La distinción entre rural y urbano ha generado un gran debate en la geografía y la sociología. Todos los planteamientos que se han ido desarrollando muestran algún problema que dificulta, en algún momento, su utilización. De ahí que cada vez más parece que se tiende a no emplear las categorías de urbano y rural, yendo al uso del concepto de continuum rural/urbano (De Block y Polansky, 2011; Millward y Spineey, 2011; Yang et al., 2011; Oliva, 1995). Pese a ese problema, consideramos que es posible intentar mostrar un enfoque sociológico diferente que podría ayudar a desbrozar el sendero. La razón de que esta diferenciación no sea tan sencilla proviene del hecho de que el concepto de urbanización no es tan evidente:

"Lo que hace sobremanera complejo el análisis y la definición de este término es la íntima conexión entre el proceso de urbanización y otros procesos de cambio, tanto en la época moderna y contemporánea, como en el pasado. Esta conexión es tal que a menudo el concepto de urbanización tiende a señalar el proceso global, es decir, a «identificarse con la totalidad de esos cambios»" (Germani, 1969: 146).

A menudo, y especialmente en la sociología, el concepto de urbanización ha englobado a todo un proceso general de cambio social, político y económico en la sociedad mal llamada «occidental». Esto contrasta con la idea más habitual de que la elevada concentración demográfica en áreas geográficas concretas es la que establece el criterio de diferenciación entre lo rural y lo urbano. Este proceso de transformación originado en la Revolución Industrial generó una serie de concepciones teóricas sobre la sociedad. De hecho, para analizar la nueva sociedad industrial y discernir sus peculiaridades se consideró necesario desarrollar mecanismos de comparación con otros tipos de sociedades, especialmente con la más diferente, con la más primitiva (Rocher, 1979). De aquí surgieron planteamientos como el de Tönnies que diferenciaba entre la comunidad y la sociedad, como el de Durkheim que estableció la distinción entre la solidaridad mecánica y la solidaridad orgánica como base de los distintos tipos de sociedades, etc.

El debate rural/urbano ha estado condicionado por la consideración de que el entorno rural es que ha evolucionado trayendo consigo la generación de lo urbano y de su cultura. Manuel Castells considera que "lo que se llama "cultura urbana» corresponde perfectamente a una cierta realidad histórica: el modo de organización social ligado a la industrialización capitalista, en particular en su fase concurrencial. Por tanto, no se define únicamente por oposición a rural, sino por un contenido específico que le es propio..." (Castells, 1976: 102). 
Hablar de lo rural y de lo urbano no quiere decir que se haga mención de espacios que tengan que ser definidos uno en contraposición del otro. Si el espacio rural lo designáramos como $A$, entonces el espacio urbano podría ser designado como $\bar{A}$ (no-A). De tal manera que $A \neq \bar{A}$ y la suma de ambos daría el espacio total habitado por los humanos. Una concepción de este tipo, tan clásica, presenta graves problemas a la hora de tener que ir al detalle. Esta es la razón básica por la que actualmente parece que existe un consenso general sobre que esta concepción tradicional no es suficiente (Armas Quintá, 2009). Esta insatisfacción conceptual ha traído consigo que, en ocasiones, se apliquen complejos y detallados criterios de delimitación entre lo que es urbe y lo que no lo es. En este sentido, Farley y colaboradores (2002) nos muestran que la ruralidad ha sido definida por el Gobierno Federal de los Estados Unidos de América a través de lo que nosotros denominaremos como metacódigo metropolitano/no-metropolitano (distinción entre metrópolis y no metrópolis). Estos autores hablan de que este tipo de delimitación conceptual ésta basado en el criterio pragmático del dato censal, estableciendo que las poblaciones edificadas de manera continua con una densidad mayor de las 50.000 personas serán consideradas espacio metropolitano y aquellas con una densidad poblacional menor serán espacio rural o no-metropolitano. Estos autores nos dicen que las áreas metropolitanas se circunscriben a regiones poblacionales que abarcan el centro de la provincia con una o varias ciudades del núcleo básico de la misma y con una densidad poblacional mayor de 50.000 personas. Farley y colaboradores (2002) nos informan, además, que el Departamento de Agricultura de E.U.A. hizo público una nueva delimitación de que lo es una zona urbana y que es una zona rural a través de los códigos RUCA, que son áreas de tránsito diario urbano-rurales (RUCA). Los códigos RUCA se basan en medidas de la urbanización, de la densidad de población y de los desplazamientos diarios. Estas zonas de tránsito buscan permitir el análisis de estos espacios híbridos en los que la idea de urbe no es funcional y la de rural tampoco.

Posteriormente, y continuando con las delimitaciones conceptuales desarrolladas en los Estados Unidos de América², comprobamos se han introducido también los conceptos de "frontera", de "distancia" y de "urbano-rural mixto" para distinguir las zonas de población rural de las urbanas. El sistema de Oregón ${ }^{3}$ para la clasificación de los Condados presenta las siguientes definiciones:

a) Zonas urbanas: Condados dentro de áreas estadísticas metropolitanas, con una población de 1.000 habitantes o más por miIla cuadrada.

b) Urbano-rural mixto: nuevas áreas urbanas con una densidad de población de 60 a 99 habitantes por milla cuadrada.

c) Rural: una población de entre 10 y 59,9 por milla cuadrada.

d) Frontera: 0,5 a 9,9 habitantes por milla cuadrado.

e) Distancia: los condados con 0.4 o menos habitantes por milla cuadrada.

Crandall y Weber (2005) han desarrollado otro esquema de categorización en el que se identifican las siguientes categorías:

2. Hemos centrado nuestro interés en algunos trabajos desarrollados en los E.U.A. por ser, a nuestro juicio, unas de las más complejas y detalladas aproximaciones al problema de delimitación urbano/rural.

3. The Association of Maternal Health Programs (2004). 
a) Área urbana: un área con 50.000 o más habitantes y las áreas circundantes dentro de 10 millas.

b) Urbano-rural: una comunidad situada a menos de 10 millas de una zona urbana, pero con fácil acceso a los servicios de salud, con calles pavimentadas y caminos.

c) Rural: una comunidad ubicada al menos a 30 millas de una comunidad urbana, con alguna actividad comercial y un acceso razonable, pero no inmediato a la atención médica.

d) Rurales aisladas: poblaciones situadas a, por lo menos, 100 millas de un comunidad de 3.000 o más personas.

e) Frontera-rural: una zona rural que está por lo menos a 75 millas de una comunidad de menos de 2000 individuos.

Este tipo de planteamientos son un enfoque de aproximación basado en los datos cuantitativos aunque también incorpora el enfoque cualitativo en lo que se refiere a la atención médica $y$, en cierto modo, tienen en cuenta las posibilidades de flujo o de traslado poblacionales. Pese a esto, la consideración de un determinado espacio como rural, urbano, etc. no tiene en consideración fenómenos provenientes de la percepción o de los imaginarios sociales. Esto que podría parecer poco relevante es lo que ha hecho que surgiese el actual "turismo rural". Por lo tanto, el ámbito rural ha dejado de ser aquel espacio de producción agroalimentaria para ser un complemento de la actividad urbana (Fundación Encuentro, 2007).

La distancia entre el mundo rural y el urbano ha decrecido fruto de la licuefacción de este último y de la cyborgización del primero. Esto parece que obliga a introducir elementos psico-sociológicos en las categorizaciones para poder así poder trabajar con estos ámbitos rururbanos que, pese a tener elementos de cualquiera de las categorías antedichas, es modificado en base a una serie de elementos imaginarios haciendo que funcione socialmente como otro. Ejemplo de esto lo podemos encontrar en multitud de poblaciones de Galicia (NO de España). Ello implica la necesidad de inclusión de información cualitativa en estas clasificaciones. En relación con el uso de datos cualitativos en la elaboración de categorías, Paniagua y Hoggart (2002) han establecido de la existencia de tres tipos de aproximaciones diferentes al problema de diferenciación entre lo rural y lo urbano: la cuantitativa, la cualitativa y el análisis de flujos.

La primera acepta que existen distintos tipos de áreas en función de sus características socio-espaciales: urbanas, residenciales, suburbanas y rurales. Para el establecimiento de estas áreas se emplean datos estadísticos y se considera que la unidad administrativa que configura es homogénea en todo el territorio. Las definiciones de base estadística, nos dicen Paniagua y Hoggart, han tenido otros tipos de enfoques: el administrativo, el relativo al área construida del municipio, el de las regiones funcionales, los de base agrícola $y_{\text {, }}$ en último lugar, los de tamaño de población o densidad. Un subgrupo específico dentro de las definiciones de esta índole son las relativas a la ocupación laboral de la población (En las localidades rurales sería, en principio, mayoritaria la actividad agraria).

Las definiciones de tipo cualitativo están basadas, según estos autores, en las percepciones y significados de la población. Es decir, lo rural y/o lo urbano será concebido de manera diferenciada en función de una serie de esquemas socialmente construidos. Este hecho hace que la delimitación entre lo rural y lo urbano se encuentre asentada en el contexto histórico particular, en la tradición de una determinada región, en los valores de sus comunidades y en las relaciones humanas existentes. Por ello, "las representaciones que se pueden hacer de lo rural tienden a ser ge- 
ográficamente diferenciadas, aunque no queden estrictamente determinadas por factores espaciales" (Paniagua y Hoggart 2002: 64).

La tercera, los enfoques de flujos, hace mención de la circulación relativamente habitual de personas entre el mundo rural y el urbano. En línea con esto se han hecho una serie de análisis sociológicos en los que se ha comprobado que existe una especie de mito de lo rural, Ilamado el idilio rural por Paniagua y Hoggart, que de manera resumida se basa en la consideración de lo rural como próximo a la naturaleza e identificado con lo saludable, con lo puro, con lo no alterado, ni contaminado en contraposición con lo urbano. En línea con esto se han expresado teóricos de la sociología como Tönnies o Simmel. El primero afirmó que el paso de una sociedad rural a una urbana significa, en cierto modo, romper los lazos intrínsecos de los grupos primarios y caminar hacia unas relaciones impersonales propias de los grupos secundarios. Simmel, por otro lado, habla de que las personas en la ciudad se ven sometidas a una estimulación frenética lo que convierte a su propio entorno en una realidad cada vez más dura para vivir. Estas consideraciones no están demasiado alejadas de esta visión del mundo rural como relativamente idílico y el espacio urbano como contraposición a éste.

Posiblemente la mejor opción que se pueda tomar a la hora de hacer una buena escala de categorías sea aquella que incorpore los tres elementos antedichos. De este modo enfocamos la estructura de análisis de un modo integracionista tal que podamos tener cierta elasticidad para poder adecuar la herramienta a nuestras propias necesidades.

\section{Material y métodos}

El presente trabajo de investigación conjuga la lógica fuzzy (Ragin, 2000 y Smithson \& Ver- kuilen, 2006; Belohlavek \& Klir, 2011 y Arfi 2010) con la observación (Guasch, 2002) y pretende ejemplificar el modelo a desarrollar centrándonos en el estudio del caso (Coller, 2005) de la población de Santa Uxía de Riveira en la provincia de A Coruña (Galicia, España). Con este enfoque metodológico logramos, a partir de un conjunto de datos empíricos observacionales, cuantificar la información cualitativa obtenida del análisis de la morfología de la población para realizar una aproximación a lo que podría ser un modelo semicuantitativo (al estar basado en información cualitativa) de análisis del proceso de gradación entre el ámbito rural y urbano.

\section{Transformación del contexto}

Actualmente la sociedad en la que vivimos se ha visto modificada con rapidez y de un modo muy profundo. De hecho, si comparamos algunos datos ofrecidos por la Encuesta sobre Tendencias Sociales realizada en 1999 con la llevada a cabo en el 2005 comprobaremos notables transformaciones en la percepción social sobre los elementos que identifican y simbolizan mejor nuestra época actual (Tezanos, 2007). En 1999 el 42,2\% de las personas encuestadas identificaban a los teléfonos móviles como el principal símbolo de nuestra época frente al $60,6 \%$ en el año 2005. Internet era un elemento identificador según el 34,1\% en 1999 y en el 2005 ascendía al 55,1\%. Por otro lado, en 1999 el $27,8 \%$ consideraban que la televisión era un elemento identificativo y el $21,3 \%$ consideraban que lo era el coche, frente al 10,6\% que tenían esa consideración de la televisión en el 2005 y el 10,3\% que la tenían sobre el coche. Estos datos nos muestran una clara modificación de la percepción de la realidad por la sociedad española actual, pasando de una visión con reminiscencias de la Revolución Industrial a una concepción propia de la Revolución Tecnológica actual. 
Lo antedicho es una muestra de los cambios sucedidos a causa del proceso de globalización y del paso de la Modernidad a la Posmodernidad $^{4}$. La Modernidad presenta las características de una sociedad dual, semiaristocrática (burguesa) y semidemocrática, con una marcada polarización en algunos ámbitos del pensamiento. De hecho es un periodo de catástrofes y de grandes esperanzas aunque también la Modernidad es un fenómeno multidimensional y complejo (Roche Cárcel, 2009). La Posmodernidad, en cambio, está marcada por un proceso de descomposición, de incertidumbre, de sucesivas crisis y de derrumbamiento. En esta época, el subjetivismo toma una importancia hasta ahora inusitada, al igual que sucede con la fragmentación de la concepción de sujeto y del mundo. Esto, unida a las sucesivas crisis y a la incertidumbre general, ha diluido las certezas tradicionales y ha generado un distanciamiento de lo real.

Dentro del proceso de la Posmodernización tiene especial relevancia el de globalización que no es otra cosa, según Giddens (2001), que aquel proceso que anula la importancia de las distancias en el espacio y las divisiones territoriales, produciendo una reorganización del tiempo, distancia y espacio de las relaciones. En Un mundo desbocado Giddens muestra la globalización, básicamente, como un efecto derivado del gran avance en las telecomunicaciones el cual ha traído consigo una audiencia global y planetaria, así como innumerables redes de intercomunicación. La globalización, al estar situada dentro de la posmodernidad, también supone una generalización de fenómenos propios de la actual realidad social tales como la psicologización y subjetivación de todas las esferas de la vida. De tal modo que la esfera privada pasa a tomar un lugar de predominio frente a la esfera pública, o lo que es lo mismo, que los intereses privados ganan una nueva dimensión y visibilidad inédita (Martelli, 2011). Este elemento de hipertrofia de lo individual condiciona la transformación de la percepción de lo rural haciendo que, por lo menos en Galicia, se tienda a migrar hacia las regiones rurales por una concepción más individualizada de la vida logrando así evitar algunas relaciones potencialmente molestas provenientes de los espacios públicos o semipúblicos. Ejemplo claro de ello, unido a otros factores tales como los económicos y medio ambientales, son los que explican el factor de la dispersión poblacional tan marcada en Galicia.

La globalización a la que estamos haciendo mención tiene su punto de encuentro en Telépolis (Echeverría, 1994), concepto semejante al de urbe global del que habló Artemio Baigorri $(1998,2001)$. En ella tienen lugar, en referencia a los tres mundos desarrollado por Popper, el tercer gran entorno que se superpone a los otros dos en los que convencionalmente nos movemos. A saber, E1 serán los diversos espacios rurales, E2 los espacios urbanos y E3 hace referencia al nuevo espacio social posibilitado por las TIC (Echeverría, 1995 y 1999). Vemos que en esta diferenciación Javier Echeverría parece que apuesta por la oposición entre rural y urbano, aunque añadiendo un tercer elemento superpuesto a ambos: E3. E1 y E2 no son excluyentes entre sí ya que en el mundo actual los espacios rurales y urbanos están imbricándose cada día a causa de los fenómenos de globalización, de migración de

4. No vamos a entrar a debatir sobre la existencia de diferenciación entre Modernidad y Posmodernidad, así como sobre la idoneidad del segundo término. En ambos polos del debate nos encontramos con personas con gran reputación y prestigio que hacen afirmaciones de gran consideración que sobrepasan, con mucho, los intereses de este texto. Por nuestra parte asumiremos la distinción desde una perspectiva pragmática con la intención de mejorar la comprensión. 
las urbes a lo rural, de la contraurbanización y de la cyborgización de lo rural.

Lo relevante de la propuesta de Echeverría (1994, 1995 y 1999), a nuestro juicio, (aunque no excesivamente novedoso ya que, como hemos dicho, Baigorri lo desarrolla desde otro enfoque) es que E3 engloba a los demás entornos y que, además, presenta unas características muy diferentes a los otros dos entornos: es distal, reticular, electrónico, digital, representacional, multicrónico, transterritorial, asentado en el aire, etc. Dicho de otro modo, E3 presenta nítidamente los caracteres propios de la posmodernidad, de la licuefacción expuesta por Bauman y de los procesos de evanescencia a los que hace mención Roche Cárcel. Además de esto, Echeverría afirma que la mayor parte de las actividades humanas y sociales pueden desarroIlarse en E3 ya que éste es un espacio que posibilita las acciones e interrelaciones a distancia, en red y en tiempo real o diferido. De ahí que sea posible desarrollar lo que se ha denominado como sociedad de la información que se superpone a las agrícolas, urbanas, industriales, etc. Esto trae consigo el desarrollo de un nuevo entorno relacional en el que E3 obliga a que las identidades y las propias personas transiten por esta nueva sociedad con las características del sujeto posmoderno. Además, reconfiguran nuestra visión de los otros entornos haciendo que éstos no puedan ser delimitados como E1 y E2, puesto que ambos se verían modificados entre sí en el mismo E3. De tal manera que E1 y E2 pierden consistencia frente a E3 quien se convierte en la estructura vehicular de las relaciones personales y sociales. A su vez, y recordando en cierto modo lo que decíamos con anterioridad, E1 y E2 mantienen de manera residual las características de las que habla Echeverría puesto que sólo en ámbito extremos se conservan la configuración propia del mundo rural y del urbano.

Si aceptamos que el proceso de cyborgización rural se está dando, entonces comprobamos que, en la mayor parte de los casos, E1 y E2 convergen en el entorno E3. Nótese que Echeverría considera que E3 es un mundo virtual lo que podría parecer un error por nuestra parte. No es así. La cyborgización social que estamos viviendo tiene un fuerte componente virtual haciendo que los entornos tradicionales se estén virtualizando. De tal modo que sería factible modificar la designación previa y hablar de E1 y E2 como E1 3 y $\mathrm{E}_{3}$ como expresión de dos entornos cuya base común es este tercer entorno. No obstante, recuérdese que "en la Urbe Global no es tan importante la jerarquía como la dirección de los flujos (información, capital, trabajo, energía), y los roles que desempeñan los distintos nodos, pero no es menos cierto que la variedad, velocidad, grado y continuidad de la interacción social se relaciona con el tamaño, forma y función de los lugares" (Baigorri, 2003: 10).

\section{Aproximación a la lógica polivalente/fuzzy}

Este nuevo modo común de considerar el mundo rural y el urbano, y sus características nos obliga a desarrollar nuevos artefactos conceptuales que nos permitan analizar y estudiar esta realidad. Estos elementos han sido introducidos por Lofti Zadeh en 1965 quien publicó el artículo titulado "Fuzzy Sets" donde nos dice que el grado de pertenencia $\mu_{A}(x)$ de un determinado conjunto borroso tomará un valor en un intervalo cerrado $[0,1]$ en base a una función de pertenencia $f_{A}(x)$. A su vez, un subconjunto borroso A de $X$ se define mediante la aplicación $\mu_{A}: X \rightarrow$ siendo I el intervalo cerrado $[0,1]$. A su vez, un subconjunto borroso A de $X$ podrá representarse como A: $\left\{X, \mu_{A}(x)\right.$ $\mid x \in X\}$, donde $\mu_{A}: X \rightarrow I$ y $x \rightarrow \mu_{A}(x)$. Por lo tanto, cada conjunto o subconjunto borroso es vago y relativo (Kosko, 2010).

Si nos referimos al tema que aquí nos ocupa podríamos afirmar que los entornos son rurales (no-urbanos) y urbanos (no-rurales) en cierto grado. Ello es debido a que se consi- 
dera que existe un subconjunto borroso rural y un subconjunto borroso urbano pertenecientes al conjunto borroso de los núcleos poblacionales humanos. La concepción clásica consideraba que los conceptos de rural y urbano eran excluyentes, es decir que $A \vee \bar{A}$ o lo que es lo mismo que o se da $A$ o se da $\bar{A}$. No obstante, gracias a las investigaciones actuales y a la asunción de la complejidad se ha hablado del continuum rural-urbano o del concepto de rurubanidad. Además de estos aspectos epistémicos, el pensamiento difuso (fuzzy thinking) al que hacen mención Winter y Kron (2009) -y que será el que guíe nuestros pasos en este trabajo- nos conduce a una nueva visión del mundo que se centra no sólo en las bivalencias sino en las polivalencias. Por esta razón, estos autores apuestas por la utilización del tetralema taoísta como contraposición a la visión aristotélica del mundo. Es decir, enfrentan la idea de o bien $A$ o bien $\bar{A} ;$ a la de $A, A$ o $\bar{A} ; A$ y $\bar{A} ;$ y $\bar{A}$.

Este planteamiento difuso está relacionado con el razonamiento humano ordinario. De tal manera que se asumen como parte del sistema de referencia epistémico los apelativos borrosos del tipo: mucho, poco, bastante, etc., siempre y cuando estas designaciones reciban un valor cuantitativo que pueda ser utilizado por el investigador. No obstante, esta configuración teórica no elimina la objetividad sino que la asume en un contexto difuso. Por esta razón, los términos de mucho, poco, etc. tomarán una serie de valores dentro del conjunto difuso $[0,1]$ lo que permitirá movernos con cierta soltura dentro del conjunto borroso de lo rural y de lo urbano sin necesidad de establecer categorías cerradas que, en la mayoría de los casos, resultan ineficaces. En un rango de tipo $[0,1]$, algo es poco, por ejemplo, verdadero cuando toma el valor 0.1 en el conjunto, incrementando su pertenencia hasta 0.3 , manteniéndola hasta 0.4 y disminuyéndola hasta 0.5 , donde empezaría a ser más 'ni verdadero ni falso' que 'poco verdadero'. A partir de 0.6 sucedería algo semejante pero en este caso sería más 'muy verdadero' que 'poco verdadero'.

Nosotros utilizaremos este enfoque lógico polivalente basándonos en elemento desarrollados por la lógica fuzzy. En base a esto más que una lógica fuzzy lo que vamos a aplicar es una lógica trivalente y, por lo tanto una lógica graduada. Empleando este tipo de aproximaciones graduadas podemos establecer las categorías numéricas que mejor nos convengan sin tener que ceñirnos a unas predeterminadas e ir aplicándolas de manera determinada en función del área que estemos analizando. En este sentido, las características que empleemos para designar un entorno como más o menos rural (por ejemplo, densidad de población, movilidad, infraestructuras, percepción social, etc.) o incluso partes de un determinado núcleo poblacional como más o menos rural, mantendrá un "puente" con el sistema a través de relaciones difusas en las que cada ámbito de estudio tomará un determinado valor que, asimismo, lo incluirá en un lugar del conjunto borroso que estemos analizando.

\section{Aplicación al caso de Santa Uxía de Riveira (A Coruña, Galicia, España)}

El Municipio de Santa Uxía de Riveira, situado en la costa occidental española y con una población de 27.504 personas (datos del INE del 01/01/2010), como muchos otros de Galicia, tiene un marcado componente intermedio entre lo rural y lo urbano en función de la zona del municipio donde nos encontremos. Esta población está localizada relativamente lejos de un gran núcleo poblacional $(\approx 70 \mathrm{Km})$, Santiago de Compostela, y con fácil acceso a un centro médico. El comportamiento de su población está marcado por una fuerte dependencia de la pesca, actividad principal de su población ya que en 
la zona se concentran una gran cantidad de empresas de la industria acuícola, y en menor medida por actividades agrícolas. En esta zona se producen actividades típicamente rurales: gallineros relativamente cerca del centro urbano, presencia de vacas o caballos pastando en los límites del gran núcleo poblacional del Municipio, venta ambulante de pescado, etc. unida a una población urbanizada y con apariencia relativamente urbana. Además, las características tampoco se adecúan a lo que Crandall y Weber (2005) denominaron urbano-rural, ni a lo que designaron como rural o como urbano. Esto problematiza esta y otras categorizaciones que se puedan llegar a realizar al respecto. Ello no quiere decir que esta aproximación analítica sea irrelevante y deba ser eliminada, al contrario es necesario tenerla en cuenta y mejorarla en la medida de lo posible. Lo que se pretende afirmar es que la idea general de un entorno rururbano (urbano-rural) es, a nuestro juicio, deficiente porque, una vez más, establece una categoría general en la que se incluyen elementos diferentes.

Para evitar este problema consideramos que los conjuntos difusos aportan un elemento de investigación y análisis muy útil (Kok et al., 2000) ya que, en base a determinados elementos y a una serie de reglas de inferencia (SI..., Y..., Y..., ENTONCES...), podremos establecer los valores difusos y determinar las reglas de consecuencia. No obstante es imprescindible establecer una descripción cuantitativa de los rangos de urbanización/ruralización que permitan, posteriormente, establecer modelos de análisis de estos entornos o microentornos. Para ello tomaremos cuatro elementos constitutivos de estos rangos al considerar que éstos son suficientes para la caracterización de dichos rangos.

Tomaremos en consideración tres elementos definitorios de la gradación urbanización/ruralización en el ámbito gallego y tomando como ejemplo el caso de Santa Uxía de Ri- veira. Estos elementos configuran un modelo preliminar que podrá ser ampliado y mejorado pero, a nuestro juicio, nos permiten dilucidas los cambios que se podrían llevar a cabo en un determinado territorio con el objeto de mejorar la situación de una determinada población, en general o en zonas particulares de la misma.

Los elementos antedichos son:

a) Habitantes: A la hora de establecer un criterio de análisis a partir de cual podamos diferenciar los niveles de ruralidad y urbanidad el factor "cantidad de habitantes" en los municipios, ayuda a delimitar, en parte, que regiones son más claramente rurales y cuales menos. En este sentido, y según los datos elaborados por el Instituto Nacional de Estadística (2010), comprobamos (Tabla 1) que de los 2.797.653 habitantes en Galicia, una buena parte viven en municipios de tamaño medio 5.001-50.000 habitantes, 534.336 viven en las capitales de provincia y 297.124 viven en poblaciones de más 100.001 habitantes que no son capitales de provincia (concretamente en Vigo que es la única población gallega con estas características). En base a estos datos es posible designar unos niveles de pertenencia basados en el razonamiento ordinario y del tipo: muchos, bastantes, pocos y muy pocos. Estos niveles de pertenencia pueden combinarse permitiendo establecer los rangos de ruralización o urbanización que hemos expuesto anteriormente. Estos grados de pertenencia estarán establecidos en base a las reglas de inferencia subsiguientes. De tal modo que SI una población A (sea cual fuese) adopta un determinado valor de pertenencia, y una vez combinado este nivel de pertenencia con los otros, ENTONCES A estará dentro del ámbito de la ruralidad o de la urbanidad. En el caso de Santa Uxía de Riveira, comprobamos que esta zona se encuentra dentro de un nivel poblacional medio ya que el Municipio de Santa Uxía de Riveira tiene una población de 27.504 personas. 
Tabla 1. Población por tamaño del municipio y sexo

Table 1. Population by size of municipality and sex

\begin{tabular}{llll}
\hline \multicolumn{4}{c}{ Unidades: Personas } \\
\hline & Ambos sexos & Varones & Mujeres \\
\hline Total & 2.797 .653 & 1.350 .547 & 1.447 .106 \\
Capital & 534.336 & 248.910 & 285.426 \\
M. no capital. Menos de 101 hab. & 0 & 0 & 0 \\
M. no capital. De 101 a 500 hab. & 654 & 332 & 322 \\
M. no capital. De 501 a 1.000 hab. & 15.857 & 7.890 & 7.967 \\
M. no capital. De 1.001 a 2.000 hab. & 108.510 & 53.918 & 54.592 \\
M. no capital. De 2.001 a 5.000 hab. & 345.115 & 169.749 & 175.366 \\
M. no capital. De 5.001 a 10.000 hab. & 400.961 & 196.083 & 204.878 \\
M. no capital. De 10.001 a 20.000 hab. & 499.370 & 244.187 & 255.183 \\
M. no capital. De 20.001 a 50.000 hab. & 427.264 & 209.084 & 218.180 \\
M. no capital. De 50.001 a 100.000 hab. & 168.462 & 78.747 & 89.715 \\
M. no capital. De 100.001 a 500.000 hab. & 297.124 & 141.647 & 155.47 \\
\hline
\end{tabular}

Fuente: Instituto Nacional de Estadística.

b) Movilidad: El factor movilidad también tiene importancia en la diferenciación entre lo rural y lo urbano. De hecho, dado los cambios en el ámbito urbano a los que hemos hecho mención anteriormente (contraurbanización, desconcentración, etc.) se ha producido un incremento sustancial de la movilidad urbana. Ello se ha convertido en uno de los elementos más significativos de la transformación urbana derivada, entre otros factores, de un uso más extensivo del territorio habitado (García Palomares, 2008; Lizárraga, 2006; Muñoz, 2006; entre otros). En el mundo rural más extremo los niveles de movilidad son bajos y se van incrementando paulatinamente a medida que se incrementa en nivel de urbanización de las poblaciones humanas ya que las zonas más urbanizadas tienden a tener una mayor tasa de población y a cubrir un mayor espacio geográfico, obligando a la población a incrementar su movilidad diaria y el tipo de transporte utilizado. Los niveles de movilidad están vinculados, fundamentalmente, a factores comerciales, a factores relativos a la distancia entre la vivienda y el trabajo, así como a las necesidades culturales de la población. Pues bien, en el caso de este factor vemos que una población A, SI tiene unos determinados niveles de movilidad (elevada, intermedia, reducida), ENTONCES tendrá un mayor grado de la ruralidad o de la urbanidad en función de los factores a los que acabamos de hacer mención. En el caso de Santa Uxía de Riveira comprobamos -a través de la información facilitada por el Concello de Riveira- que su población presenta, como medio de transporte público, la línea de autobús entre San- 
tiago y esta población, la línea Noia-Riveira, Corrubedo-Riveira y Pobra de Caramiñal-Riveira lo que limita las posibilidades de la población ya que todas estas poblaciones están muy cerca de esta población. No obstante, y pese a estas limitaciones la movilidad entre estas zonas no es muy baja, lo que hace que nuevamente Riveira presente unas características intermedias de urbanidad en relación con la movilidad en transporte público. Si nos fijamos ahora en las posibilidades de movilidad en coches particulares comprobamos que el parque automovilístico, en Octubre del 2011 y según datos de la Dirección General de Tráfico, asciende a un total de 15.379. Esto contrasta con los 143.031 de la ciudad de A Coruña, o los 58.875 de Santiago de Compostela. A todo esto debemos añadir las posibilidades de movilidad que ofrece la presencia de la Autovía del Barbanza y que conecta Santa Uxía de Riveira con la población de Padrón y con la Autopista Vigo-Coruña. Por lo tanto, nuevamente Riveira presenta unas características generales intermedias de rururbanidad.

c) Cyborgización: En Coca y Valero (2012) se establecen unos elementos teóricos básicos a partir de los cuales es posible comprender que se quiere decir al hablar de los procesos de cyborgización del mundo rural y urbano. En líneas generales podemos decir que dicho concepto resume el proceso de artefactualización tecnocientífica de estos entornos humanos. Existe, por tanto, cierta correlación (ello no quiere decir que sean lo mismo) entre la concepción clásica de urbanización y la cyborgización. Una zona estará más cyborgizada cuando sus elementos tengan una mayor alteración tecnocientífica: antenas, semáforos, utilización de edificios como elementos de márquetin, etc. De tal modo que, a modo de ejemplo, podemos afirmar que los edificios estarán más cyborgizados cuando sean empleados como estructuras comerciales (bajos comerciales, carteles, etc.) que cuando no lo son. A su vez, las aceras más cyborgizadas serán aquellas que presenten artefac- tos tales como semáforos, papeleras, bolardos, etc. Por otro lado, los parques y jardines también presentan diferente grado de cyborgización en base a la alteración tecnocientífica que presenten (uso de organismos vegetales modificados, configuración estética, etc.). En definitiva, la cyborgización de las poblaciones humanas tiene mucho que ver con el desarrollo tecnocientífico y comercial de las mismas y con la configuración de estas como partes de un entramado fundamentalmente tecnológico vinculado a las actividades cotidianas de las personas. Es decir, si en una determinada población nos encontramos con una zona en la que están colocados aerogeneradores ello no quiere decir que esta población esté cyborgizada ya que éstos apenas modifican su vida. Ahora bien, si lo que tenemos son campos con cultivos biotecnológicos o agroenergéticos la cosa cambia, ya que estos sí condicionan el quehacer diario. Si nos fijamos ahora en el caso de Santa Uxía de Riveira comprobamos que el nivel de cyborgización de esta población es bajo. De hecho, la calle más cyborgizada es la Avenida del Malecón (Imagen 1) cuyo nivel de alteración varía en función de la zona donde nos encontremos de dicha avenida.

Una vez explicados los tres elementos básicos del modelo se hace necesario establecer un conjunto de reglas de inferencia condicionales que reflejarán los mecanismos subyacentes a los posibles escenarios cualitativos que nos podamos encontrar (Kok et al. 2000). Dichas reglas serán la base de los elementos causales que se ponen en juego en el modelo que estamos proponiendo para el caso de Santa Uxía de Riveira. De tal modo que SI la población tiene una determinada característica numérica, Y la movilidad otra, Y la cyborgización otra, ENTONCES el grado de urbanización/cyborgización será $X$. Por lo tanto es perfectamente posible establecer una regla de inferencia teórica posibles de este fenómeno de gradación (tabla 2). La regla general sería algo así como: $P \& M \& C \rightarrow(R / U)$. 

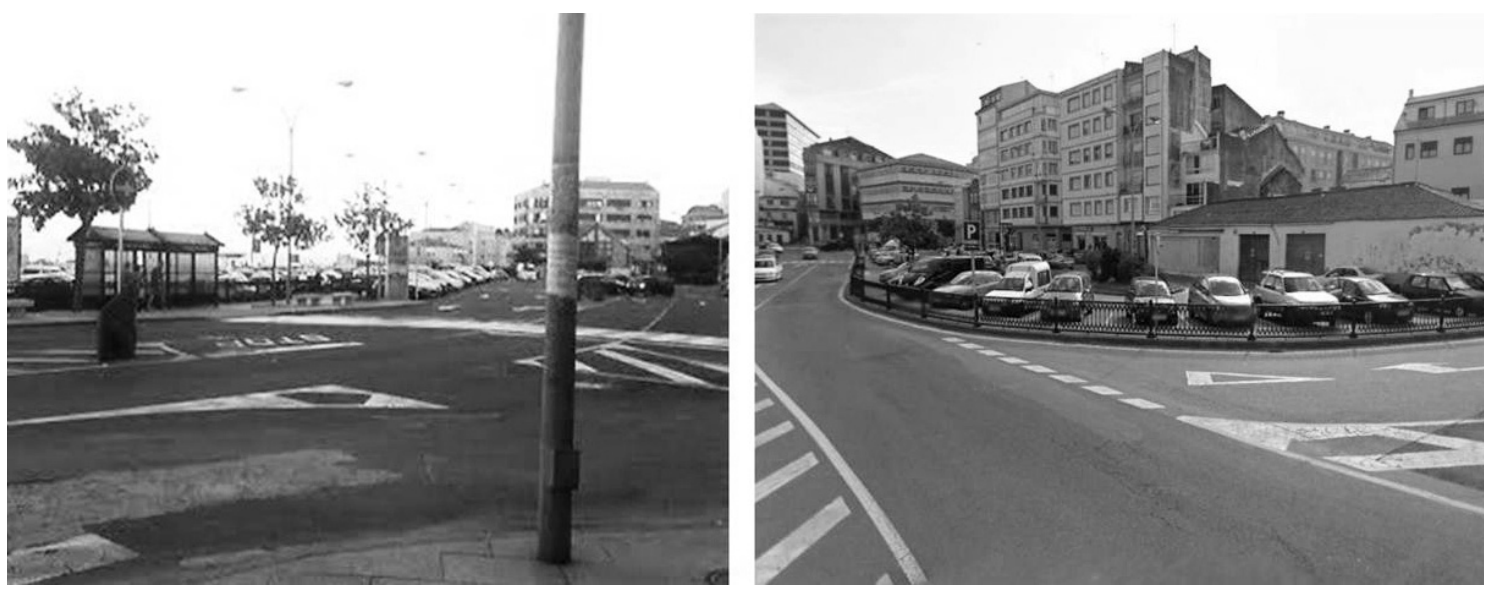

Imagen 1. Vista de la Avenida del Malecón.

Imagen 1. View of the Malecon Avenue.

Fuente: Imágenes de Ribeira (año 2010) en la que se constatan los grados de cyborgización de la misma calle. Izquierda, zona más cyborgizada. Derecha, zona menos alterada.

Cada uno de los tres factores (población, movilidad y cyborgización), dada su difusividad, constituyen un conjunto difuso $(1,2$ y 3 , respectivamente) en los cuales, en función de la zona o población considerada, tendrá un valor de pertenencia determinado que podrá ser cuantificado según la pertenencia que dicha región tenga en este conjunto. Ejemplifiquemos esto.

Las zonas de la imagen aérea de Santa Uxía de Riveira marcadas con una flecha son zonas con muy baja cyborgización (recuérdese que son zonas de cultivo insertas en el tejido urbano). Por lo tanto, estas zonas tomarán un valor cuantitativo de 0,25 (baja cyborgización) en el conjunto difuso $\mathrm{m}_{\mathrm{c}}$. En el lado opuesto nos encontramos el centro del núcleo poblacional el cual presenta un nivel de cyborgización bastante alto pero sin llegar a ser muy alto. Entonces, el grado de pertenencia de esta zona al conjunto difuso $\mathrm{m}_{\mathrm{c}}$ será de 0,75 . Téngase en cuenta que el nivel de cyborgización es difícil de cuantificar y comporta un factor de subjetividad, de ahí que hallamos optamos por la designación de dos niveles de cyborgización. Además, consideramos que el valor 1,00 no podrá ser alcanzado, puesto que constantemente sucederán procesos de alteración tecnológica que implican que nunca se alcanzarán grados de pertenencia extremos en este conjunto.

Para ver cómo funciona el modelo que estamos proponiendo tomaremos ahora una imagen más general de Santa Uxía de Riveira (Imagen 3) y estableceremos tres grandes zonas del núcleo poblacional (zona A, B y C). Cada una de ellas presenta características diferentes que harán que tengan distinto grado de pertenencia en cada uno de los conjuntos difusos establecidos.

La teoría de los conjuntos difusos incluye operaciones de intersección, complemento e inclusión. De ahí que la unión difusa de dos conjuntos $(X \cup Y)$ es definida como el grado máximo de pertenencia en el conjunto en cuestión: $m_{X \cup Y}=\max \left(m_{X}, m_{Y}\right)$. A su vez, estos mismos conjuntos pueden intersectarse de manera difusa $(\mathrm{X} \cap \mathrm{Y})$, de tal modo que la pertenencia de esta intersección difusa (fuzzy) 
Tabla 2. Reglas de inferencias posibles del fenómeno de gradación urbano/rural Table 2. Rules of possible inference of the urban/rural gradation phenomenon

\begin{tabular}{|c|c|c|c|c|}
\hline Regla & Población $\left(f_{1}\right)$ & Movilidad $\left(f_{2}\right)$ & Cyborgización $\left(f_{3}\right)$ & $\begin{array}{l}\text { Grado de } \\
\text { rurbanización }\end{array}$ \\
\hline 1 & Alta & Elevada & Alta & Tipo $1_{C}$ \\
\hline 2 & Alta & Elevada & Baja & Tipo $1_{\text {B }}$ \\
\hline 3 & Alta & Intermedia & Alta & Tipo ${ }^{1} \mathrm{C}$ \\
\hline 4 & Alta & Intermedia & Baja & Tipo $1_{\text {B }}$ \\
\hline 5 & Alta & Reducida & Alta & Tipo $1_{\mathrm{A}}$ \\
\hline 6 & Alta & Reducida & Baja & Improbable \\
\hline 7 & Media & Elevada & Alta & Tipo $2_{c}$ \\
\hline 8 & Media & Elevada & Baja & Tipo $2_{\text {B }}$ \\
\hline 9 & Media & Intermedia & Alta & Tipo $2_{c}$ \\
\hline 10 & Media & Intermedia & Baja & Tipo $2_{\text {B }}$ \\
\hline 11 & Media & Reducida & Alta & Tipo $2_{A}$ \\
\hline 12 & Media & Reducida & Baja & Improbable \\
\hline 13 & Pequeña & Elevada & Alta & Tipo $3_{A}$ \\
\hline 14 & Pequeña & Elevada & Baja & Tipo $3_{A}$ \\
\hline 15 & Pequeña & Intermedia & Alta & Tipo $3_{B}$ \\
\hline 16 & Pequeña & Intermedia & Baja & Tipo $3_{\text {B }}$ \\
\hline 17 & Pequeña & Reducida & Alta & Tipo $3_{C}$ \\
\hline 18 & Pequeña & Reducida & Baja & Tipo $3_{C}$ \\
\hline
\end{tabular}

Fuente: Elaboración propia.

es definida como el grado mínimo de pertenencia en el conjunto: $m_{X \cap Y}=\min \left(m_{X}, m_{Y}\right)$. Por otro lado es necesario mostrar que las tres regiones ( $A, B$ y $C$ ) muestras tres grados diferentes de rururbanidad. De tal modo que podemos considerar a cada una de éstas como un conjunto difuso: $m_{A}, m_{B}$ y $m_{C}$. Como hemos dicho anteriormente cada conjunto difuso $\left(m_{X}\right)$ tomará un determinado valor dentro del conjunto "universal" $U$, tal que $U$ $=\left\{m_{A}, m_{B}\right.$ y $\left.m_{C}\right\}$. Asimismo, cada conjunto $m_{X}$ tendrá una serie de subconjuntos 1, 2 y 3 que coinciden con los factores población, movilidad y cyborgización. Además, y por otro lado, es necesario tener en cuenta que la consideración de las reglas de inferencia que hemos establecido previamente nos indican que, SI se una determinada zona (sea la que sea) tiene una población concreta, $Y$ la movilidad es una determinada, Y la cyborgización es alta o baja, ENTONCES el grado de urbanización/ruralización será uno u otro. En este sentido, a partir de la tabla 2 donde quedan establecidas 18 reglas de inferencia que delimitan el modelo 


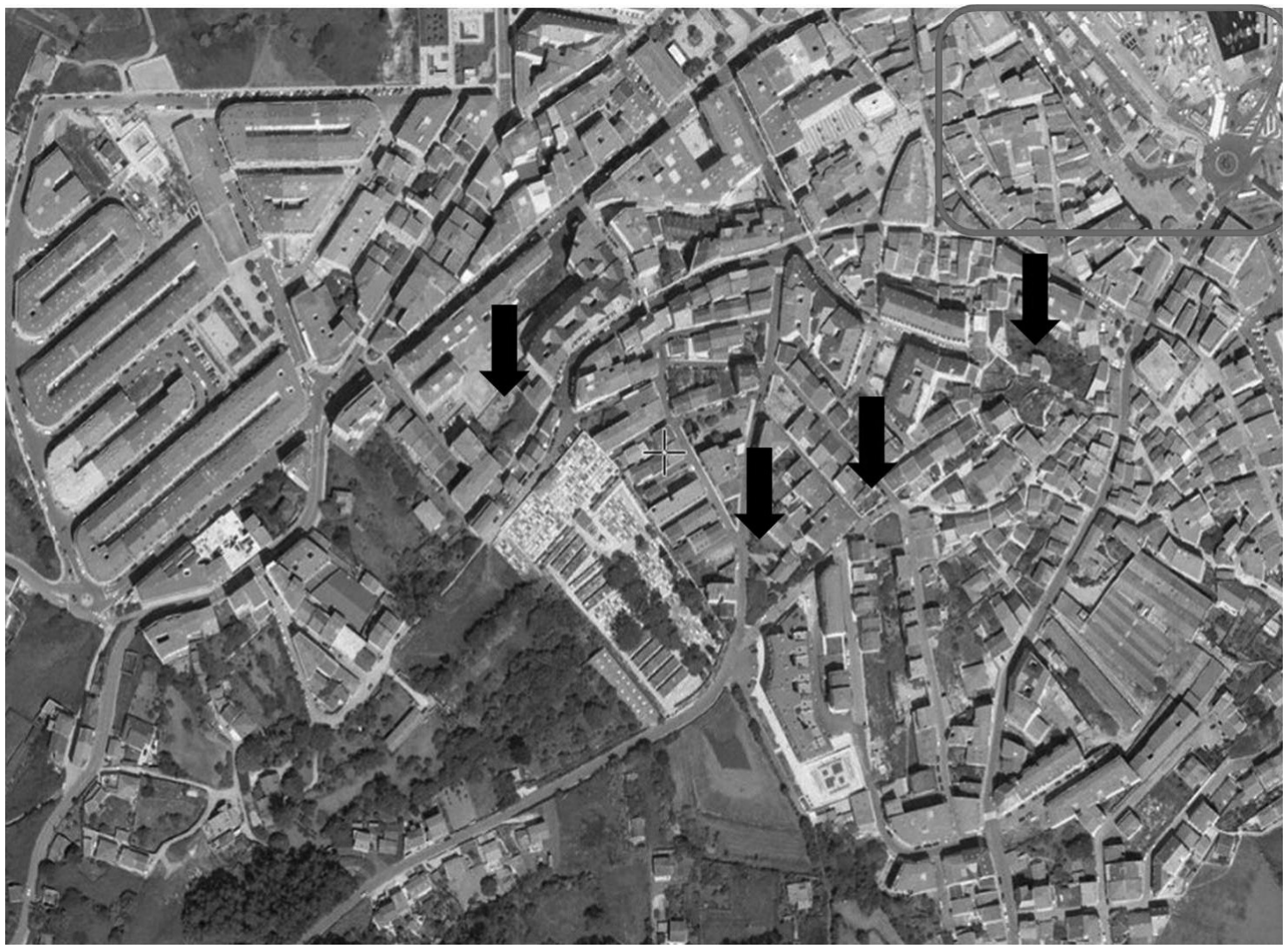

Imagen 2. Vista aérea de Santa Uxía de Riveira (Nivel 17).

Imagen 2. Aerean view of Santa Uxía de Riveira (Level 17).

Fuente: Visor Six Pac de la Xunta de Galicia. Las flechas indican algunas de las zonas dedicadas a la agricultura y el cuadro la zona de mayor relevancia de la población.

operacional que estamos desarrollando. Estas 18 reglas de inferencia son todas aquellas posibles que podrían llegar a darse en función de este modelo. No obstante, ello no quiere decir que todas ellas se pongan en juego en todos los casos, sino que en función de la población que estemos investigando unas u otras serán las que tengamos que tener presentes.

En el caso de la ciudad de Santa Uxía de Riveira (cuya población es menor que la del Municipio) nos encontramos con un problema añadido a la hora de contabilizar la población de las zonas de estudio puesto que muchos de los hogares habitados no constan en el censo de oficial. En base a esto hemos optado por un cálculo aproximado de la población (en base al censo y a la visita personal a los diferentes edificios) tomando como grado de pertenencia máximo la zona más poblada (zona C: alrededor de las 3.000 personas, zona B: alrededor de las 2.000 personas, zona A: alrededor de las 1.500 personas). Con respecto a $f_{2}$ hemos optado por considerar el acceso que la población tiene a los diferentes medios de transporte público, 


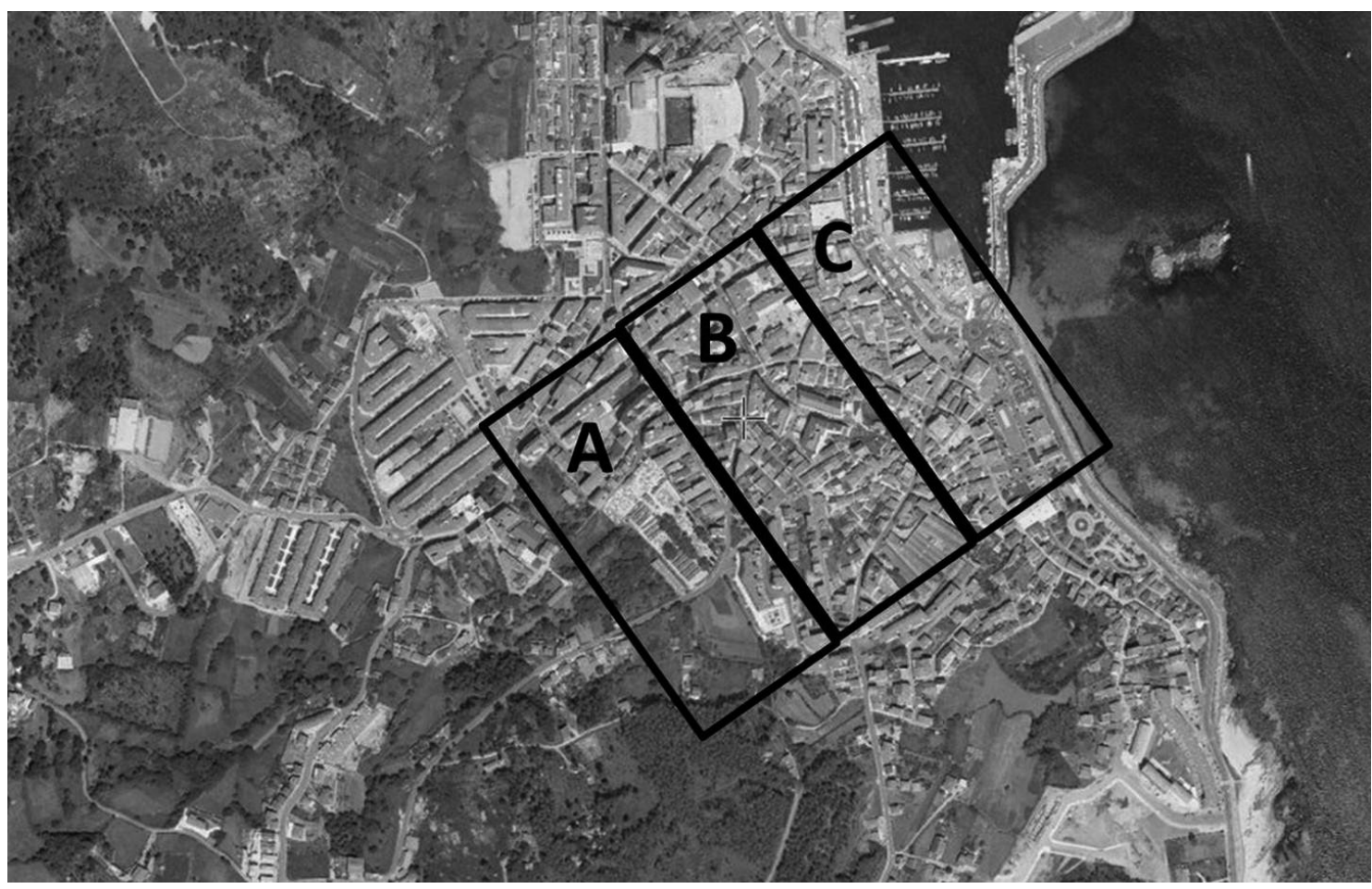

Imagen 3. Visión aérea de Santa Uxía de Ribeira (Nivel 16). Imagen 3. Aerean view of Santa Uxía de Ribeira (Level 16).

Fuente: Visor Six Pac de la Xunta de Galicia. En la imagen establecemos tres regiones con diferentes grados de desarrollo urbano/rural siendo la A la más rural y menor urbana, B la intermedia y C la más urbana y menos rural.

así como a las principales vías de comunicación. De ahí las zonas con mayor grado de pertenencia al conjunto serán aquellas mejor comunicadas. En este sentido hemos podido comprobar que las zonas C y B son las más próximas tanto a la estación de autobuses, como a las paradas de los taxis. No obstante las dos vías principales de comunicación con la población están situadas, una en la zona del malecón y otra en la zona A. Por último, a la hora de analizar $f_{3}$ también hemos decidido considerar que la zona con mayor grado de pertenencia será la que tenga mayor nivel de cyborgización. En este caso el análisis es menos problemático al quedar perfectamente patente el impacto tecnocientífico en las tres áreas (ver Tabla 2).

Gracias a esta modelización preliminar vemos que la discrepancia entre conjunción $\left(f_{1} \cup f_{2}\right.$ $\left.\cup f_{3}\right)$ y la disyunción $\left(f_{1} \cap f_{2} \cap f_{3}\right)$ nos permite conocer la urbanización o ruralización de las zonas de estudio. En nuestro caso una gran discrepancia nos permite afirmar la necesidad de desarrollar mecanismos de compensación de la rururbanización de esta zona. En la zona B la discrepancia es menor y en $\mathrm{C}$ el nivel de urbanización es alto. 
Tabla 3. Conjuntos difusos urbano/rurales en Santa Uxía de Riveira (A Coruña, Galicia) Table 3. Urban/rural fuzzy sets of Santa Uxía Riveira (A Coruña, Galicia)

\begin{tabular}{cccccc}
\hline Zonas & Factor $1\left(f_{1}\right)$ & Factor $2\left(f_{2}\right)$ & Factor $3\left(f_{3}\right)$ & $f_{1} \cup f_{2} \cup f_{3}$ & $f_{1} \cup f_{2} \cup f_{3}$ \\
\hline A & 0,50 & 0,75 & 0,25 & 0,75 & 0,25 \\
B & 0,75 & 0,50 & 0,25 & 0,75 & 0,50 \\
C & 1,00 & 1,00 & 0,75 & 1,00 & 0,75 \\
\hline
\end{tabular}

Fuente: Elaboración propia. Se muestra el valor que toman los factores $f$ (1: población, 2: movilidad, 3: cyborgización) en cada una de las regiones (A, B y C), así como el dato de la operación de unión (U) e intersección $(\cap)$.

\section{Conclusiones}

En base a esta información y a las reglas de inferencia podemos establecer que Santa Uxía de Riveira tiene unas necesidades altas de incremento de la movilidad sobre todo en su zona $B$ y de aumento en los niveles de cyborgización de A y B. De hecho es necesario aumentar los niveles de alteración tecnológica a través de una descentralización de la zona comercial. Por último vemos, y esto es lo más relevante en esta ocasión, que el grado de ruralización es relativamente alto. Ello supone la necesidad de desarrollar mecanismos de descentralización del área comercial de esta población e implementar la comunicación y el transporte en las vías secundarias de acceso. Estas conclusiones, como es obvio, deberían ser tenidas en cuenta por las autoridades competentes para producir un incremento en el grado de urbanización de esta región haciendo que esta población pase a tener un mayor crecimiento como supuesta ciudad que es.

A través del presente trabajo de investigación hemos propuesto y puesto en funcionamiento una aproximación a un modelo preliminar de análisis y categorización de la gradación entre la ruralidad y urbanidad. Se han detectado tres factores fundamentales que entran en juego en la diferenciación en- tre lo rural y lo urbano. Gracias a este modelo podemos ver cuáles son las acciones generales que una población, como puede ser Santa Uxía de Riveira, necesita para implementar su desarrollo. Como ya hemos dicho, este modelo es preliminar y es susceptible de mejora. De hecho, los autores del texto somos conscientes de la complejidad de lo social aunque consideramos que también necesitamos modelos de análisis que nos permitan simplificar los problemas y poder facilitar así la labor política, económica, legislativa, etc. Por otro lado también consideramos es necesario incrementar la profundidad del mismo mejorando su aplicabilidad a las áreas antedichas.

Actualmente nuestro grupo de investigación está trabajando en ello y tiene intención de mejorar todos estos aspectos. No obstante, consideramos que a través del estudio del caso propuesto hemos mostrado la viabilidad del mismo y su posible potencial.

\section{Bibliografía}

Arfi B, 2010. Lingüistic fuzzy logic in social Science. Springer, London.

Armas Quintá FJ, 2009. Sociedade da información e desenvolvemento rural. Análise de novos procesos sociais e territoriais em rexións periféricas. 
O caso de Galicia [CD]. Servizo de Publicacións Universidade de Santiago, Santiago de Compostela.

Baigorri A, 1995. Del urbanismo multidisciplinario a la Urbanística transdisciplinaria. Una perspectiva sociológica. Ciudad y Territorio/Estudios Territoriales III, 104: 313-328.

Baigorri A, 1998. De la tierra ignota al jardín terrenal. Transformaciones en los usos y funciones del territorio en la urbe global. Ciudades 4: 149-164.

Baigorri A, 2001. Hacia la urbe global. Badajoz, mesópolis transfronteriza. Editora Regional de Extremadura, Mérida.

Baigorri A, 2003. Urbanismo y urbanistas en la urbe global. Conferencia impartida en el Seminario: Urbanismo: ¿cambios o permanencias?, Escuela Interdisciplinar de Postgrados, Facultad de Artes, Universidad Nacional de Colombia, Bogotá (Agosto, 2003). Disponible en: http://www.eweb.unex.es/eweb/sociolog/BAlGORRI/papers/urbanistas \%20y\%20urbe\%20glo bal.pdf (10 de octubre de 2012).

Belohlavek R \& Klir G, 2011. Concepts and fuzzy logic. MIT Press, New York.

Berry BJL, 1976. The counterurbanization process: urban America since 1970. In: Berry BJL (Ed.), Urbanization and counterurbanization, Urban Affairs Annual Reviews 11, Sage, London: 17-30 pp.

Bryant CR, Russwurm LH \& McLellan AG, 1982. The City's Countryside. Land and management in the rural-urban fringe. Longman, Nueva York.

Capel H, 1975. La definición de lo urbano. Estudios Geográficos 138-139, febrero-mayo: 265-301.

Castells M, 1976. La cuestión urbana. Siglo XXI, Madrid.

Coca JR y Valero Matas JA, 2012. Theoretical reflexions on the rural cyborgical development in Spain. Between the utopy and the social imaginaries. Lurralde 35: 53-66.

Coller X, 2005. Estudio de casos, Cuadernos Metodológicos $n^{\circ} 30$. CIS Madrid.

Crandall M \& Weber B, 2005. Defining rural Oregon: an exploration. Rural Studies Program, 13. Oregon State University, Oregon.
De Block G \& Polasky J, 2011. Light raiways and the rural-urban continuum: technology, space and society in late nineteenth-century Belgium. Journal of Historical Geography 37, 3: 312-328.

Durkheim E, 2001. La division social del trabajo. Akal, Madrid.

Echeverría J, 1994. Telépolis. Destino, Barcelona.

Echeverría J, 1995. Cosmopolitas domésticos. Anagrama, Barcelona.

Echeverría J, 1999. Los señores del aire. Telépolis y el tercer entorno. Destino, Barcelona.

Farley DO, Shugarman LR, Moira Inkelas PT, Ashwood JS, Zeng F \& Harris KM, 2002. Trends in Special Medicare Payments and Service Utilization for Rural Areas in the 1990s, MR-1595CMS. RAND Corporation, Santa Monica (Californi). Disponible en: http://www.rand.org/ pubs/monograph_reports/MR1595 (20 de Junio de 2011).

Ferrás Sexto C, 2000a. Ciudad dispersa, aldea virtual y revolución tecnológica. Reflexión acerca de sus relaciones y significado social. Scripta nova. Revista Electrónica de Geografía y Ciencias Sociales 69, 68. Disponible en: http://www.ub.edu/ geocrit/sn-69-68.htm (04 de Octubre de 2011).

Ferrás Sexto C, 2000b. Counterurbanization and Common Agricultural Policy. Implications for the Galician Country. Internacional Colloquium New Urban and New Rural Pattern. Consejo de Europa, Asamblea Parlamentaria, Strasbourg.

Ferrater Mora J, 1998. De la materia a la razón. Alianza, Madrid.

Fundación Encuentro, 2007. Informe España 2007 una interpretación de su realidad social. Fundación Encuentro-CECS, Madrid.

García Palomares JC, 2008. Incidencia en la movilidad de los principales factores de un modelo metropolitano cambiante. EURE (Santiago) 34, 101: 5-24.

Germani G, 1969. Sociología de la modernización. Paidós, Buenos Aires.

Giddens A, 2000. Un mundo desbocado. Los efectos de la globalización en nuestras vidas. Taurus, Madrid. 
Giddens A, 2001. Medios de Comunicación y cultura popular. Alianza, Madrid.

Guasch O, 2002. Observación participante, Cuadernos Metodológicos $n^{\circ} 20$. CIS, Madrid.

Kok JL, Titus M \& Wind HG, 2000. Application of fuzzy sets and cognitive maps to incorporate social science scenarios in integrated assessment models. Integrated Assessment 1: 177-188.

Kosko B, 2010. El futuro borroso o el cielo en un chip. Drakontos-Crítica, Barcelona.

Lizárraga Mollinero C, 2006. Movilidad urbana sostenible. Un reto para las ciudades del siglo XXI. Economía, Sociedad y Territorio. VI, 22: 283-321.

Martelli CGG, 2011. O protagonismo do indivíduo na sociedade hipermoderna. Estudos Sociológicos 16, 30: 141-160.

Millward H \& Spinney J, 2011. 'Active Living' Related to the Rural-Urban Continuum: A TimeUse Perspective. Journal of Rural Health 27, 2: 141-150.

Mitchell CJA, 2004. Making sense of counterurbanization. Journal of Rural Studies 20: 15-34.

Muñoz F, 2006. El tiempo del territorio, los territorios del tiempo. En Nogué y Romero (Eds.), Las otras geografías. Crónica, Valencia: 235-254.

Oliva Serrano J, 1995. Mercados de trabajo y reestructuración rural: una aproximación al caso castellano-manchego Ministerio de Agricultura, Pesca y Alimentación, Madrid.

Paniagua Mazorra A y Hoggart K, 2002. Lo rural, ¿Hechos, discursos o representaciones? Una perspectiva geográfica de un debate clásico. ICE Revista de Economía 803, Noviembre-Diciembre: 61-71.

Ragin CC, 2000. Fuzzy-Set Social Science. Chicago University Press, Chicago/London.
Roche Cárcel JA, 2009. La sociedad evanescente. Anthropos, Barcelona.

Rocher G, 1979. Introducción a la sociología general. Herder, Barcelona.

Simmel G, 1986. El individuo y la libertad. Ensayos de crítica de la cultura. Península, Barcelona.

Smithson M \& Verkuilen J, 2006. Fuzzy Set Theory. Applications in the Social Sciences. Series: Quantitative Applications in the Social Sciences. Sage publications, California/London/New Delhi.

Tezanos JF, 2007. Los impactos sociales de la revolución tecnológica. In: Tezanos, J.F. (Ed.) Los impactos sociales de la revolución científicotecnológica. Noveno foro sobre tendencias sociales. Sistema, Madrid: 31-62.

The Association of Maternal Health Programs, 2004. From rural to remote America. Family health care in Alaska, Idaho, Oregon and Washington. The Association of Maternal Health Programs, Washington DC.

Tönnies F, 1947. Comunidad y sociedad. Losada, Buenos Aires.

Winter L \& Kron T, 2009. Fuzzy Thinking in Sociology. In: Seising, R. (Ed.) Views on Fuzzy Sets and Systems from Different Perspectives, Springer, Berlin: 301-320.

Yang TC, Jensen L \& Murali H, 2011. Social Capital and Human Mortality: Explaining the Rural Paradox with County-Level Mortality Data. Rural Sociology 76, 3: 347-374.

Zadeh L, 1965. Fuzzy Sets. Information and Control 8: 338-353.

(Aceptado para publicación el 14 de noviembre de 2012) 